\title{
Aportes significativos del proceso de intervención comunitaria con la Escuela Popular de Comunicación Alternativa Jaime Garzón de la ciudad de Cúcuta, Colombia
}

\author{
Carlos Lasso-Urbano \\ Magister en Ciencias Sociales. Trabajador social \\ Universidad Mariana. San Juan de Pasto, Colombia \\ https:/ / orcid.org/0000-0001-5407-1953・1bca12123@hotmail.com
}

\section{Resumen}

El presente artículo es el resultado de la sistematización de experiencias que se hace del proceso de intervención comunitaria desarrollado con los integrantes de la Escuela Popular de Comunicación Alternativa Jaime Garzón de la ciudad de Cúcuta, Colombia, durante el año 2019 y primer semestre del 2020, en donde se logra aportar estratégicamente desde el Trabajo Social a potenciar la organización comunitaria por medio del fortalecimiento del diálogo, las relaciones interpersonales, el trabajo colectivo y la formación en comunicación popular.

Palabras clave: Intervención comunitaria; Acción colectiva; Comunicación popular; Organización comunitaria; Participación; Sistematización de experiencias; Trabajo colectivo; Trabajo Social.

Recibido: 28/07/2020 | Aprobado: 10/11/2020 | Publicado: 01/01/2021

(c) (1) (2) Esta obra está bajo una Licencia Creative Commons Atribución-NoComercialCompartirIgual 4.0 Internacional.

Financiación o proveniencia del artículo: Artículo derivado del proyecto de sistematización "Las preprácticas en el área de Trabajo Social con Comunidades y Organizaciones Sociales, una apuesta a la praxis profesional", avalado por la Universidad Simón Bolívar, sede Cúcuta, realizado en el periodo agosto de 2019, junio de 2020.

\section{¿Cómo citar este artículo? / How to quote this article?}

Carlos Lasso-Urbano, C. (2021). Aportes significativos del proceso de intervención comunitaria con la Escuela Popular de Comunicación Alternativa Jaime Garzón de la ciudad de Cúcuta, Colombia. Prospectiva. Revista de Trabajo Social e intervención social, (31), 259-279. doi: 10.25100/ prts.v0i31.10467. 
Lasso-Urbano

\title{
Significant Contributions of the Community Intervention Process with the "Escuela Popular de Comunicación Alternativa Jaime Garzón" in the City of Cúcuta, Colombia
}

\begin{abstract}
This article is the result of the systematization of experiences of the community intervention process developed with the members of the Popular School of Alternative Communication Jaime Garzón in the city of Cúcuta, Colombia, during 2019 and the first semester of 2020, where it is possible to contribute strategically from Social Work to strengthen community organization through the strengthening of dialogue, interpersonal relations, collective work and training in popular communication.

Keywords: Community intervention; Collective action; Popular communication; Community organization; Participation; Systematization of experiences; Collective work; Social work.

Sumario: 1. Introducción, 2. Metodología, 3. Contextualización de la Escuela Popular de Comunicación Alternativa Jaime Garzón, 4. El proceso de intervención, 4.1. Inicio del proceso de intervención, 4.2. Las estrategias de acción, 5. Hallazgos, 6. Conclusiones, 7. Referencias bibliográficas.
\end{abstract}




\section{Introducción}

Hay que producir condiciones en que sea posible aprender críticamente... que seamos creadores, instigadores, inquietos, rigurosamente curiosos, humildes y persistentes. La curiosidad, como inquietud indagadora, como búsqueda de esclarecimiento, forma parte integrante del fenómeno de la vida. No habría creatividad sin la curiosidad que nos mueve y nos pone pacientemente impacientes ante el mundo que no hicimos. (Freire, 2004)

Apostarle a la producción de conocimiento desde la intervención social, constituye un desafío permanente para Trabajo Social como profesión, pero también una necesidad insoslayable para quienes estamos desde la academia llevando a cabo procesos con comunidades y organizaciones sociales. En el trabajo comunitario es tan fundamental que se conozca el territorio, su complejidad, la forma en que los individuos intercambian ideas, las costumbres, la experiencia real de los sujetos, para así comprender sus dinámicas y particularidades, y no desde el sentido común, desde acercamientos fugaces, efímeros e incluso inexistentes sobre la realidad concreta. De manera que la batalla contra el sentido común y el empirismo ingenuo debe darse desde el acercamiento real hacia los sujetos de intervención, "para lograr ver y hablar del mundo tal cual es, hay que aceptar estar siempre en lo complicado, lo confuso, lo impuro, lo vago, etc., e ir así contra la idea común del rigor intelectual" (Bourdieu, 2002, p. 13), por lo tanto, el investigador y/o profesional social debe tener la capacidad de fecundar el espíritu del trabajo comunitario hasta llegar a conseguir el proceso del autoconocimiento, superándose de ese modo, la contemplación ${ }^{1}$. Esa es la principal apuesta a la que se pretende contribuir con el presente documento.

Las experiencias son procesos históricos y sociales dinámicos, aquí entran también en juego otra serie de elementos que es fundamental tener en cuenta, como es el territorio en el que se desenvuelven, las situaciones que las hacen posibles, las percepciones e interpretaciones que se hagan de estas y las relaciones que se establecen entre los individuos; he ahí la importancia de entenderlas, visibilizar y compartir sus enseñanzas para poder comunicarlas y esto se logra estando ahí, haciendo parte de la vida y cotidianidad con las comunidades. Para reflexionar sobre esas experiencias debemos comenzar por sistematizarlas pues la sistematización por ser "una construcción colectiva de conocimientos sobre las prácticas para transformarlas (Torres-Carrillo, 2011, p. 48), produce conocimientos y aprendizajes significativos que posibilitan el apropiarse de su sentido, abordarlas conceptualmente y orientarlas hacia el futuro con una perspectiva transformadora y emancipadora.

La interpretación crítica en el proceso de sistematización, solamente se puede concebir como completa, si logra convertirse en práctica transformadora, de manera que "la

1 Trascender la contemplación se constituye en asunto determinante para dejar de lado la forma abstracta e ideal de ver la realidad y en ese sentido, avanzar hacia la articulación consciente del pensamiento y la acción, donde no sólo como individuos, sino principalmente desde lo colectivo y/o gremial se logre superar aquella concepción que ha terminado reduciéndola nada más a una actividad utilitaria y de corte individual 
sistematización de experiencias debe cerrar siempre cada ciclo de su espiral, con un retorno a la práctica, enriquecido con los elementos teóricos. Por eso también, el proceso de sistematización es en sí mismo- un proceso de educación popular" (Jara-Holliday, 1998, p. 5); en efecto, el ejercicio consiste en abordar la experiencia, mediante la reconstrucción de los saberes producidos en una práctica concreta, siendo pensada con y para las comunidades, en un ejercicio que va desde la experiencia a la teoría y desde la teoría a la experiencia, en un juego dialéctico permanente. Recuperar y reflexionar sobre la intervención comunitaria desarrollada con la Escuela Popular de Comunicación Alternativa Jaime Garzón de la ciudad de Cúcuta, es una forma de apostarle a la visibilización del proceso social y comunitario, comprenderlo ampliamente y reconstruir reflexivamente la acción profesional, en un contexto de contemporaneidad:

Tenemos muchos aprendizajes en nuestra práctica, pero no los reconocemos, se nos van olvidando en el camino porque caemos en el activismo y perdemos la posibilidad de parar un momento para recopilar, para recoger y para reflexionar en torno a todos esos aprendizajes los cuales, por tanto, muchas veces se pierden para nosotros mismos y por tanto no podemos compartirlos con otras personas. (Jara-Holliday, 2018, p. 17)

Algunos de los ejes que ameritan ser sistematizados, son los procesos educativos y organizativos de carácter popular, aquellos que surgen de las dinámicas económicas, políticas, sociales y culturales en las que se encuentran inmersos los sectores urbano populares, para así rescatar y compartir sus formas de organización, la construcción de propuestas alternativas que derivan de la perspectiva popular. Es desde estas temáticas que se plantea la necesidad de elaborar reflexiones conceptuales y metodológicas a partir de los aprendizajes de la experiencia con el fin de avanzar en la gestación de estrategias educativas y comunicativas que aporten al quehacer de las/los trabajadores sociales.

Es pertinente y necesario para los profesionales reflexionar sobre la práctica, desde la propia experiencia, para generar un saber real, pues tal como señala Sánchez-Vásquez (2011) "La teoría necesita de la práctica no sólo para surgir y verificarse en ella, sino también para formarse "de una manera definitiva" (p. 212). La teorización debe presentarse en función de la práctica y no desligada de ésta, sino por el contrario, en completa unidad, teniendo en cuenta que la praxis es conocimiento teórico, pero también es práctica, que integra la actividad subjetiva y objetiva²; la teorización por sí sola no es praxis, mientras que la teoría permanezca en su estado puramente teórico no se transita de ella a la praxis, quizás pueda transformar la conciencia que tiene el hombre sobre los hechos, sus ideas respecto a las cosas, pero en ningún momento logrará transformar las cosas mismas.

2 Desde Sánchez-Vásquez (2003) la praxis se entiende como aquella crítica de la realidad concreta, pero también la autocrítica. Concibiéndose como una actividad práctica que hace y rehace cosas, siendo a la vez algo más que práctica o su unidad con la teoría, pues es una práctica que aspira radicalmente a mejorar una sociedad. Praxis es trabajo y el trabajo es la unión entre la idea y el acto concreto. 
Para el desarrollo del presente artículo, se partirá por comprender la realidad, desde las personas que integran la Escuela Popular de Comunicación Alternativa Jaime Garzón, entendiendo sus formas de vida, sus pensamientos, su cultura, el contexto que habitan y todos los demás elementos característicos de este territorio periférico de la ciudad de Cúcuta. Aquí se hace necesario dilucidar el desarrollo de la experiencia en el marco de la producción material de la sociedad, a partir de la realidad existente, trascender hacia la comprensión de la misma práctica, lo que implica, además, transformar experiencias en conceptos, es decir, llegar a la verdadera praxis. En un primer momento se realiza la presentación de la metodología y la contextualización de lo que es la Escuela Jaime Garzón, destacando que se direccionó desde el enfoque Histórico-Dialéctico; posteriormente, se describe la forma en que se adelantó el proceso de intervención social en barrio Minuto de Dios; más adelante, se exponen las estrategias de acción implementadas desde lo profesional; luego, se continúa con la presentación los hallazgos, a través de la interpretación crítica de la experiencia; por último, se presentan las conclusiones a las que se llega en el marco de este ejercicio profesional.

\section{Metodología}

La presente sistematización de experiencias obedece al proceso de intervención social desarrollado con los integrantes de la Escuela Popular de Comunicación Alternativa Jaime Garzón, de la ciudad de Cúcuta. Las fuentes primarias para la recolección de la información son los diferentes jóvenes que hicieron parte de este proceso, así que se tuvieron en cuenta sus diálogos y relatos (recogidos a través de entrevistas, conversaciones informales), sus comentarios y también sus reflexiones. Las fuentes secundarias fueron los diarios de campo, diarios de notas, videos, fotografías, que sin duda fueron determinantes para profundizar en la recuperación de la experiencia de este proceso de intervención. La sistematización se aborda desde el enfoque Histórico-Dialéctico, dado que como manifiesta Jara-Holliday (1994), las experiencias deben entenderse como un aspecto fundamental de la práctica social, que se caracteriza por ser histórica, contradictoria, compleja y dinámica, atravesadas por unas relaciones de poder, de allí su importancia de comprenderlas dialécticamente.

Una vez se organiza la experiencia en sus diversos momentos, integrando además aspectos objetivos y subjetivos de la misma, los cuales son fundamentales para su comprensión, análisis e interpretación, se da paso al análisis de los factores que garantizaron el proceso y se realiza la reconstrucción de la experiencia, definiéndose para ello la consulta de textos teóricos para el abordaje de las categorías de análisis, de igual manera se prosiguió a la construcción del objetivo de la sistematización, la hipótesis de acción y el eje de sistematización los cuales se abordan a continuación. 


\section{Plan de sistematización}

\section{Objetivo de la sistematización}

Comprender el sentido y los aportes del proceso de intervención social llevado a cabo con los integrantes de la Escuela Popular de Comunicación Alternativa Jaime Garzón de la ciudad de Cúcuta con el fin de fortalecer la organización comunitaria y los vínculos intergrupales.

\section{Hipótesis de Acción}

Si se genera un proceso de fortalecimiento del diálogo y el trabajo colectivo entre los integrantes de la Escuela Popular de Comunicación Alternativa Jaime Garzón de la ciudad de Cúcuta, se emprenderán estrategias colectivas de organización comunitaria.

\section{Eje de la sistematización}

¿Cómo se genera un proceso de intervención social que contribuya a potenciar la organización comunitaria entre los integrantes de la Escuela Popular de Comunicación Alternativa Jaime Garzón de la ciudad de Cúcuta, a partir del fortalecimiento del diálogo y el trabajo colectivo?

\section{Contextualización de la Escuela Popular de Comunicación} Alternativa Jaime Garzón

En el año 2011 en San José de Cúcuta se crea la Escuela Popular de Comunicación Alternativa Jaime Garzón, caracterizada por ser un proceso comunitario de comunicación popular que comienza a tener presencia en algunos barrios periféricos de la ciudad, con el fin de gestar procesos educativos y comunicacionales que aporten al fortalecimiento de la participación y a la organización barrial en aras de promover el ejercicio de la ciudadanía crítica, teniéndose en cuenta la realidad social, política y económica tan compleja de la capital norte santandereana. En los nueve años que ya tiene el proceso, sus integrantes han visto la necesidad permanente de potenciar la formación y fortalecer varios aspectos como colectivo, para posesionar su trabajo desde las diversas áreas desde las que se orienta su accionar político.

El colectivo comunicacional actualmente lo integran en su mayoría jóvenes, entre ellos migrantes venezolanos retornados, también víctimas del conflicto social, político y armado; gran parte de sus integrantes, pertenecientes a familias de bajos recursos económicos. De acuerdo con las directrices de la escuela, entre los objetivos que orientan su trabajo colectivo 
Lasso-Urbano

se encuentran: contribuir a formar sujetos críticos, con capacidad de analizar el contexto de la región a través de la comunicación popular; formar líderes propositivos y personas que cuenten con la capacidad de construir su proyecto de vida, un proyecto que esté lejos de la violencia y de la falta de oportunidades sociales que tienen en su entorno, muchos de los integrantes consideran a la escuela como una familia, pues siempre han contado con el apoyo de ésta, en especial en los momentos más difíciles.

La Escuela Jaime Garzón maneja dos líneas de actuación, una transversal y otra acumulativa; la primera es la línea de comunicación, la cual se lleva a cabo a partir de la formación política que se realiza por medio de capacitaciones relacionadas con el manejo de cámara fotográfica y de video, la creación de programas de radio y del periódico popular, lo anterior se implementa en algunos colegios y en los barrios donde estos están ubicados. La segunda línea es la deportiva, con la cual se brinda una opción a los jóvenes de los barrios para que practiquen un deporte como el fútbol, debido a que por la situación económica no pueden acceder a escuelas deportivas por el alto costo de la mensualidad. El Atlético Garzón (equipo de fútbol de la escuela), no solo busca brindar esta opción a mujeres y hombres jóvenes, sino también quiere impulsar el liderazgo, es decir, que en unos años sean ellos y ellas los que puedan replicar esta propuesta en otros territorios, para así poder aumentar el número de población beneficiada, y utilizarla además como estrategia para alejar a los jóvenes de la violencia y del consumo de sustancias psicoactivas.

En el 2020, el proceso cuenta con la participación de aproximadamente 70 jóvenes; cabe mencionar que en ciertas temporadas del año este número es cambiante, debido a las dinámicas del territorio y del individuo que a veces afronta el bajo nivel académico, la crisis económica y los problemas familiares. Sobre este último, se puede decir que la mayoría de los jóvenes tienen en común que no cuentan con un núcleo familiar estable, además, en diferentes hogares, las madres son cabeza de hogar. Es importante señalar que algunos de sus integrantes son estudiantes universitarios, especialmente de programas de comunicación social en la universidad Francisco de Paula Santander y en la universidad de Pamplona.

La escuela cuenta en este momento con procesos organizativos en las comunas siete (barrio Chapinero), ocho (barrio Minuto de Dios, Nuevo Horizonte), comuna nueve (barrios Loma de Bolívar y Los Alpes) y en el mega colegio La Pastora que se ubica entre los barrios La Pastora y El Rodeo; es de resaltar que la comuna ocho o Juan de Atalaya, tiene como organismos aliados a las Juntas de Acción Comunal de estos barrios; asimismo, vienen articulando procesos con la Casa Morada y la Corporación Red Departamental de Derechos Humanos del Norte de Santander. Generalmente las reuniones o encuentros con los jóvenes líderes se realizan en las instalaciones de estas dos últimas instituciones. 
Lasso-Urbano

Como proceso popular expresan que también poseen debilidades, la principal es la económica, ya que se necesitan recursos para el desarrollo de las dinámicas propias del colectivo, desde el transporte de los jóvenes, la obtención de herramientas de trabajo como cámaras, micrófonos, grabadoras, balones de fútbol, conos, platillos, entre otros; la principal herramienta con que cuentan para visibilizar y difundir el trabajo colectivo que realizan es la página web y las redes sociales, donde tienen cerca de tres mil seguidores, destacándose además, que mediante dichas páginas se encargan de difundir constantemente noticias que consideran son invisibilizadas en los medios masivos de comunicación. Habitualmente los jóvenes participan de diversas actividades de organización y movilización que se desarrollan en Cúcuta, tal como se evidencia en la siguiente figura.

Figura 1. Participación de la Escuela Jaime Garzón en la movilización del 1 de mayo en la ciudad de Cúcuta, mayo de 2019.

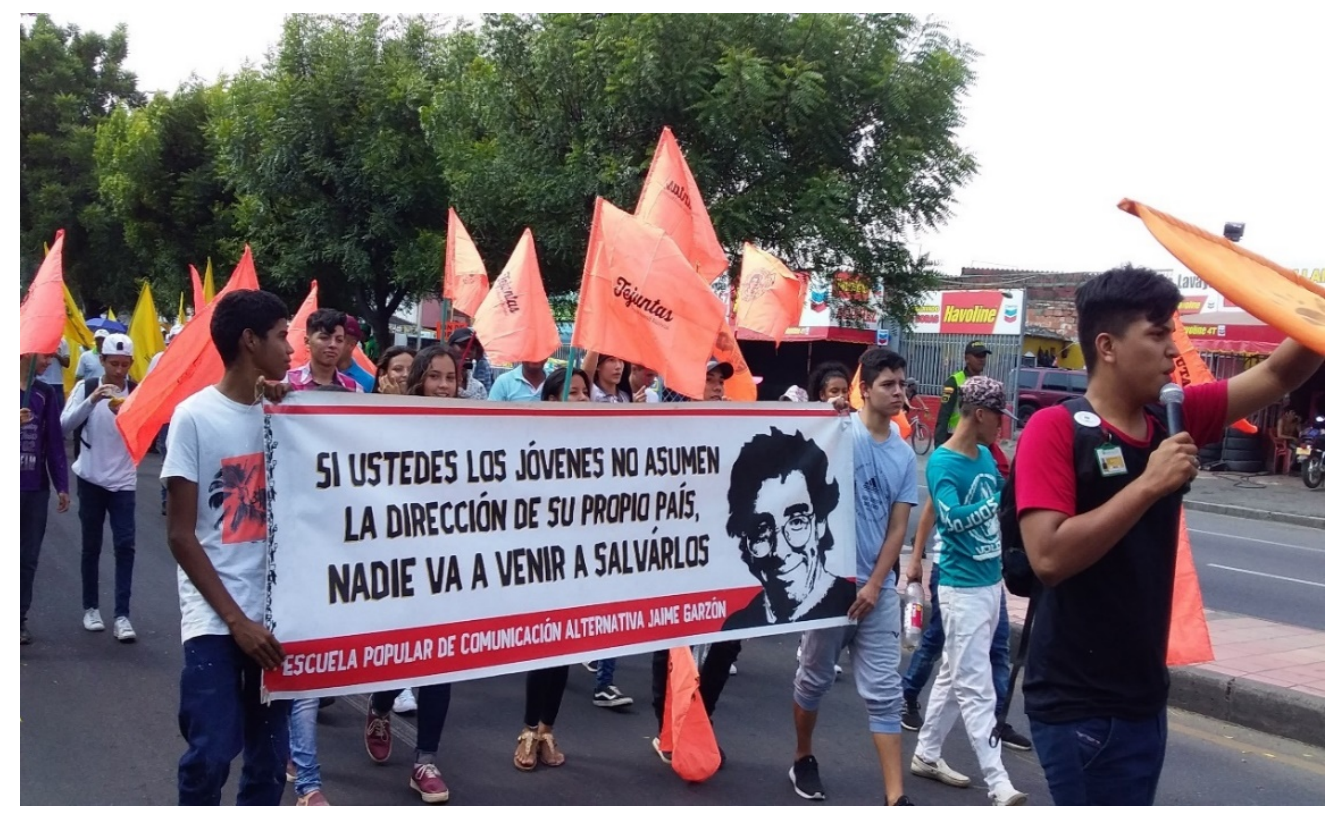

Fuente: elaboración propia.

\section{El proceso de intervención}

\subsection{El inicio del proceso de intervención}

En el mes de enero de 2019, comienzo a acercarme de forma más amplia a la Escuela Popular de Comunicación Alternativa Jaime Garzón, en especial al proceso que llevan a cabo en el barrio Minuto de Dios, caracterizado por ser el que mayor participación de jóvenes tiene a nivel de barrios en los que hacen presencia; yo había tenido la oportunidad de conocer el proyecto comunicacional hace varios años cuando aún era estudiante universitario, para ese momento, apenas estaban proyectándose como colectivo en la 
Lasso-Urbano

ciudad. Empiezo a leer acerca de qué es la Escuela, respecto al trabajo organizativo que adelantan en barrios populares de la ciudad, logro identificar que sus integrantes en su mayoría son jóvenes entre los 14 y 23 años de edad, gran parte de ellos ya con varios años haciendo parte del proceso.

Los primeros acercamientos con algunos de sus líderes se desarrollaron cerca de las instalaciones de la universidad Simón Bolívar; ahí aproveché para presentarme, contarles quién era yo, sobre mi interés por conocer y participar en el proyecto comunicacional no solamente como persona, sino también como docente de Trabajo Social. Ellos, de igual forma, se presentaron y hablaron respecto a la Escuela Jaime Garzón, en la cual participan hace ya bastantes años. Con el fin de que conozca más a profundidad el proceso, me facilitaron documentos y material audiovisual, en ese momento aproveché para expresarles mi interés por aportar y aprender de la propuesta, aspecto que fue muy bien visto por parte de ellos y ellas.

Para entender más a profundidad el proceso, necesitaba hacer el acercamiento y reconocimiento de la realidad concreta de las comunidades donde éste se gestaba, con sus problemáticas, recorrer el barrio, observar sus particularidades, dialogar con la gente, así que después de un par de reuniones, luego de la invitación hecha por sus líderes a participar en una actividad que tenían programada en las instalaciones de la Junta de Acción Comunal del barrio Minuto de Dios, decido aceptar. Allí logro conocer el territorio, a gran parte de sus integrantes, ellos y ellas me acompañaron a hacer el recorrido por sitios estratégicos del sector. Describían de forma muy detallada los problemas sociales, políticos y económicos presentes en la zona, los conocían perfectamente, pues llevaban años conviviendo con ellos y con la ausencia histórica del Estado. Luego de varias visitas al barrio, de establecer una relación más cercana con estos jóvenes, de escucharlos sobre su experiencia en la Escuela Jaime Garzón, logro identificar que se presentan relaciones conflictivas entre algunos de sus integrantes, además, que existe una limitación en términos de equipos para la producción audiovisual y para el desarrollo mismo de su trabajo colectivo.

Los días domingos los distribuían por lo general en diversas actividades colectivas, por una parte, se organizaban para la venta de comidas rápidas, especialmente de perros calientes, era la forma en que lograban obtener dinero para suplir los gastos que tenían como escuela; por otro lado, cada tres semanas se reunían en la biblioteca municipal de Cúcuta para la realización de sus respectivas jornadas de estudio, es decir, para formarse políticamente. Resultaba bastante gratificante ver a jóvenes pensándose su país, pero también con la necesidad de cualificarse, de comprender conceptualmente el porqué de tantas injusticias y desigualdades sociales en Colombia, en el departamento Norte de Santander y, máxime, en el contexto donde habitan. 


\subsection{Las estrategias de acción}

Una vez conocí a profundidad la dinámica de la Escuela Jaime Garzón, tanto por medio de la observación participante como a través de entrevistas informales y diálogo de saberes, identifico que además de las capacidades, tienen, una serie de problemáticas o aspectos que para ese momento considero necesario enfrentar e intervenir para así fortalecer aún más la dinámica del proceso y el trabajo colectivo, por lo que se ve la necesidad de idear estrategias de acción que trasciendan la inmediatez, que se piensen a mediano y largo plazo para ser implementadas de forma participativa con la gente; acá es donde entiendo la importancia de comenzar a vincular a la universidad Simón Bolívar a este proyecto para así poner la institución al servicio de la comunidad, de manera que se comienza a entablar alianzas con instancias de la universidad que aportarían considerablemente, entre ellas, Pedagogía, Extensión y el programa de Trabajo Social.

Entre las necesidades identificadas estaba la baja cualificación teórica-metodológica en comunicación popular, lo que hacía que en muchas ocasiones se cayese de forma inconsciente en el simple activismo e incluso, en el amarillismo ${ }^{3}$, o se reprodujeran acciones de los medios masivos de comunicación; también existían relaciones conflictivas entre algunos de sus integrantes tal como se señaló líneas arriba, lo que afectaba el trabajo colectivo, el diálogo y la propia dinámica grupal; en los hogares de varios de los jóvenes los conflictos familiares son una constante, por lo que se veían afectados emocionalmente, incluso algunos optaban por irse de sus casas o por desertar de su formación escolar, etc., a su vez, el entorno adquiría también niveles de complejidad por el conflicto social, político y armado en el cual se encuentra inmerso este barrio periférico de la ciudad de Cúcuta.

Las necesidades y capacidades identificadas fueron socializadas con el colectivo por medio de un encuentro intergrupal. Ellos estuvieron de acuerdo en dar respuestas a dichas necesidades por medio de un plan de acción que integraba tres líneas principalmente: la formación teórico-metodológica, la organización y por último, las relaciones interpersonales y el trabajo colectivo, cada una de las mismas direccionada desde el trabajo comunitario. Para avanzar con el fortalecimiento del proyecto, en primer lugar, se definieron varios encuentros de formación en comunicación popular, donde se contó con el acompañamiento de estudiantes de últimos semestres de comunicación social de las universidades de Pamplona y Francisco de Paula Santander a quienes algunos de los referentes de la Escuela Jaime Garzón invitaron a aportar con sus conocimientos.

3 Cuando hablo del simple activismo me estoy refiriendo a llevar a cabo actividades solamente por hacer, sin que exista reflexión respecto a las mismas, frente al contexto, dejando de lado el aspecto conceptual; con respecto al amarillismo, lo que hacían era caer en el sensacionalismo, presentar noticias donde aspectos como la sangre, la violencia eran lo llamativo para ellos, no aquello que originaba dichas problemáticas. 
Lasso-Urbano

De igual manera se aprovechó que la Universidad Simón Bolívar había invitado a la ciudad a dos investigadores desde España, los señores Marc Pallares Piquer, doctor en Pedagogía y Comunicación Audiovisual de la Universidad de Valencia, profesor de la Universidad de La Rioja, y José Gavaldá Roca, profesor de comunicación audiovisual de la Universidad de Valencia, para la realización de un taller formativo en el barrio Minuto de Dios con los integrantes de la Escuela Popular de Comunicación Alternativa Jaime Garzón. La gestión se hizo a través de la oficina de Pedagogía de la Universidad, sin duda cada una de estas actividades fueron esenciales para potenciar lo teórico-metodológico de la comunicación popular. Por mi parte, también continué apoyando la formación (dada mi experiencia en la construcción de este tipo de comunicación), mediante encuentros desarrollados con los integrantes de la escuela a lo largo del año 2019 donde poco a poco se fueron visibilizando la apropiación y los aprendizajes adquiridos tanto de forma individual como colectiva. En la figura que aparece a continuación se puede evidenciar una de las sesiones formativas llevadas a cabo con los integrantes de la Escuela Jaime Garzón.

Figura 2. Taller de formación en edición audiovisual.

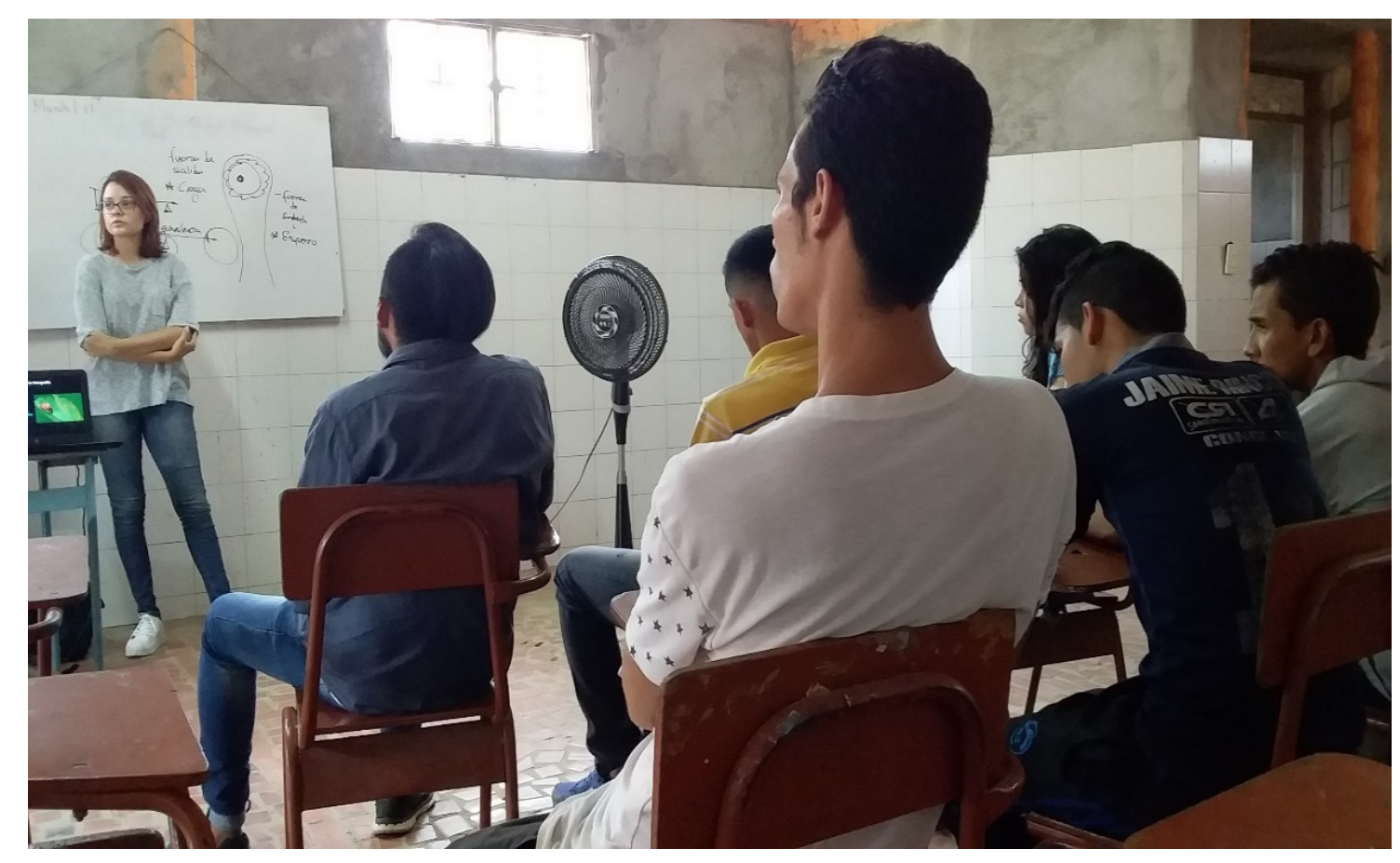

Fuente: elaboración propia.

A partir de las discusiones generadas desde los espacios de formación, de los avances obtenidos hasta el momento, surgió la propuesta de crear en conjunto con el semillero de investigación que yo coordinaba para ese momento en la universidad Simón Bolívar, el programa radial "Hablemos de la comuna", por medio del cual se busca visibilizar las 
problemáticas sociales, políticas y económicas presentes en los barrios populares de la ciudad, esas que son invisibilizados por los medios de masas 4 .

También se pretendía resaltar las capacidades colectivas de la gente, en aras de dar a entender que en la periferia no todo son problemas, este programa se convertía en la oportunidad real para que los sujetos sean parte activa del proceso de construcción del mensaje y del producto comunicacional, teníamos muy claro que la comunicación popular se constituía no solamente en proceso educativo-comunicativo, sino además, en estrategia comunitaria para fortalecer la organización y participación de las comunidades y de la misma escuela; cada fin de semana, con cámaras, teléfonos celulares y grabadoras en mano, nos dirigíamos a barrios periféricos para la construcción del respectivo programa, entre estos barrios se encuentran Minuto de Dios, La Libertad, La Loma de Bolívar, entre otros, con lo que fuimos despertando el interés en los ciudadanos y ciudadanas del común por hacer parte y verse representados en el mismo; un ejemplo de ello es la figura que se presenta a continuación, donde los habitantes de uno de los barrios periféricos de la ciudad participan en la grabación del programa radial.

Figura 3. Grabación programa radial "Hablemos de la comuna” en el barrio La Loma de Bolívar, ciudad de Cúcuta.

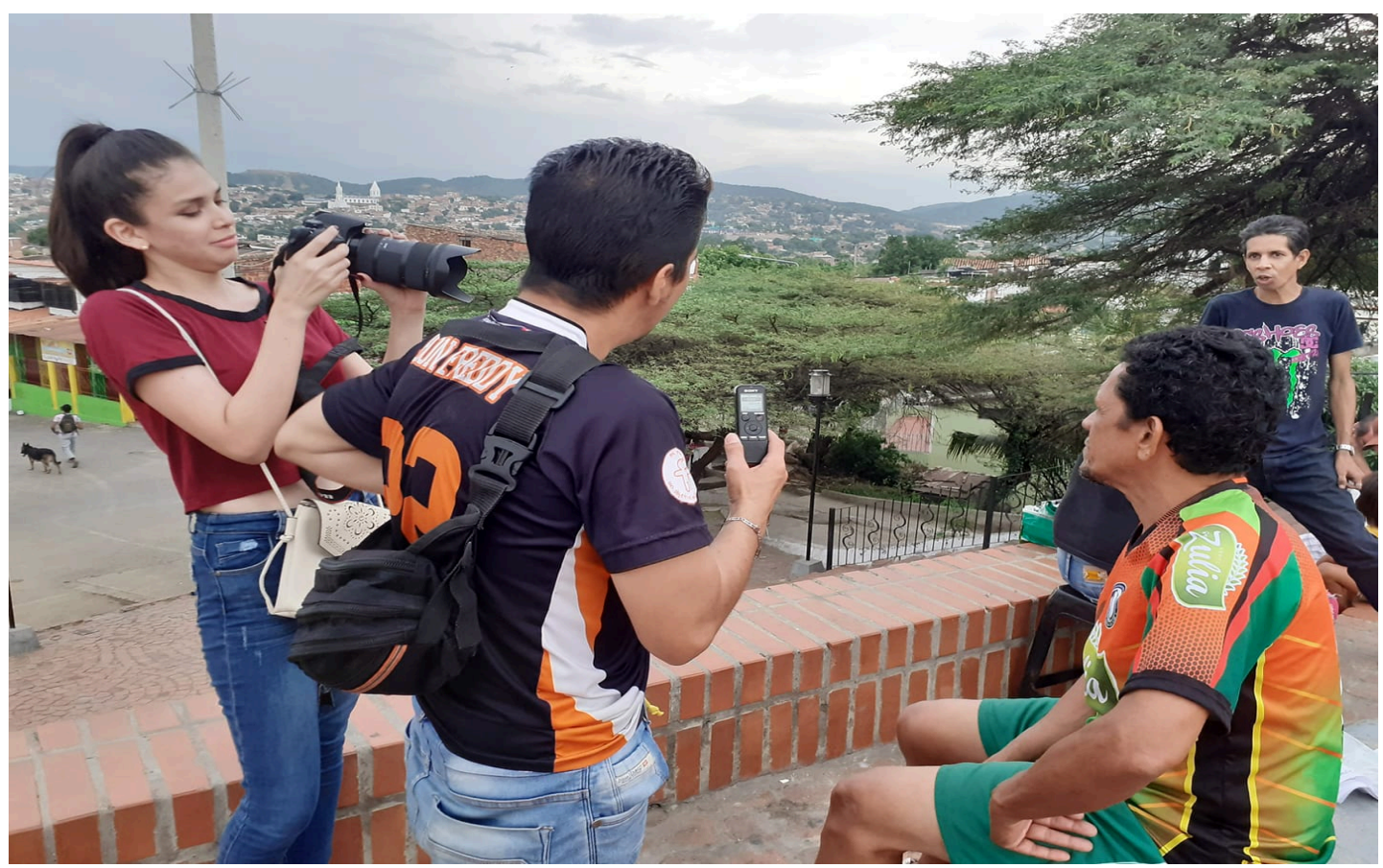

Fuente: elaboración propia.

4 Fue una estrategia que permitió también a su vez potenciar el trabajo en equipo, especialmente entre los integrantes de la Escuela Popular de Comunicación Alternativa Jaime Garzón. 
En cuanto a las relaciones conflictivas, la mala convivencia, los problemas familiares, la afectación en el trabajo en equipo, sin duda constituían elementos que afectaban lo subjetivo y las relaciones interpersonales de los integrantes de la escuela. De igual forma en que lo hacían las condiciones objetivas como son la pobreza, los altos niveles de violencia presentes en el barrio, los efectos del conflicto social, político y armado. Para fortalecer el componente emocional y las relaciones interpersonales, se presentó a la oficina de extensión de la Universidad Simón Bolívar, en conjunto con el programa de Trabajo Social, el proyecto denominado "Las pre prácticas en el área de Trabajo Social con comunidades y organizaciones sociales, una apuesta por la praxis profesional". Con éste se pretendía que los estudiantes de quinto semestre, quienes estarían a mi cargo, realizaran sus procesos de intervención comunitaria en algunos barrios periféricos de Cúcuta y del municipio de Los Patios, entre estos, en el barrio Minuto de Dios, en articulación con colectivos barriales, organizaciones sociales y comunitarias entre las que se encontraba la Escuela Jaime Garzón.

El grupo de estudiantes seleccionado para llevar su proceso de intervención comunitaria con la Escuela realizó su acercamiento y reconocimiento del barrio. Luego de haber establecido confianza con los integrantes de la misma se desarrolló el diagnóstico social; a través de un grupo focal, entrevistas semiestructuradas, observación no participante y por medio de revisión documental, se identificaron las problemáticas que afectaban la dinámica grupal y familiar, así que a partir de ahí, con mi apoyo como docente del área, construyeron su plan de intervención materializado a través de un proyecto social, para dar respuesta a dichas necesidades. El proyecto lleva implementándose dos semestres (2019-2 y 2020-1) con resultados bastante significativos para la población con la que se ha desarrollado el proceso. Además de aportar al crecimiento intergrupal, durante el primer semestre del 2020 se construyó la cartilla lúdico-pedagógica denominada "Construyendo vínculos a través de la comunicación", mediante la cual se brinda herramientas participativas para el fortalecimiento del diálogo y de las relaciones interpersonales de los jóvenes, para ser utilizada por parte de la Escuela Jaime Garzón, ésta se constituyó en el producto final del semestre.

Los encuentros adelantados por parte de los estudiantes de Trabajo Social con los jóvenes pertenecientes a la Escuela Jaime Garzón e incluso con algunos integrantes de sus familias, se realizaron en tres horas semanales durante 14 semanas que es el tiempo estipulado por cada semestre; se acudió a estrategias como el fútbol para transformar la competencia por el trabajo en equipo, los insultos por el diálogo y también la importancia de la escucha y el respeto hacia el otro; es menester señalar que dos de los estudiantes que realizaron su intervención comunitaria con el grupo en el segundo semestre del 2019, terminaron integrándose a la Escuela Jaime Garzón lo que se ve como un aspecto bastante significativo, pues hoy aportan desde su formación al fortalecimiento del proyecto colectivo. El proceso ha sido clave para que las personas reconozcan la presencia de problemáticas, pero también, vean la necesidad de idear estrategias que permitan dar solución a las mismas 
Lasso-Urbano

por más difíciles que resulten. En la siguiente figura se puede evidenciar la participación de los integrantes de la Escuela en la realización de una de las actividades de los estudiantes de Trabajo Social.

Figura 4. Actividad “Reconociendo las problemáticas presentes en el entorno" (18-102019).

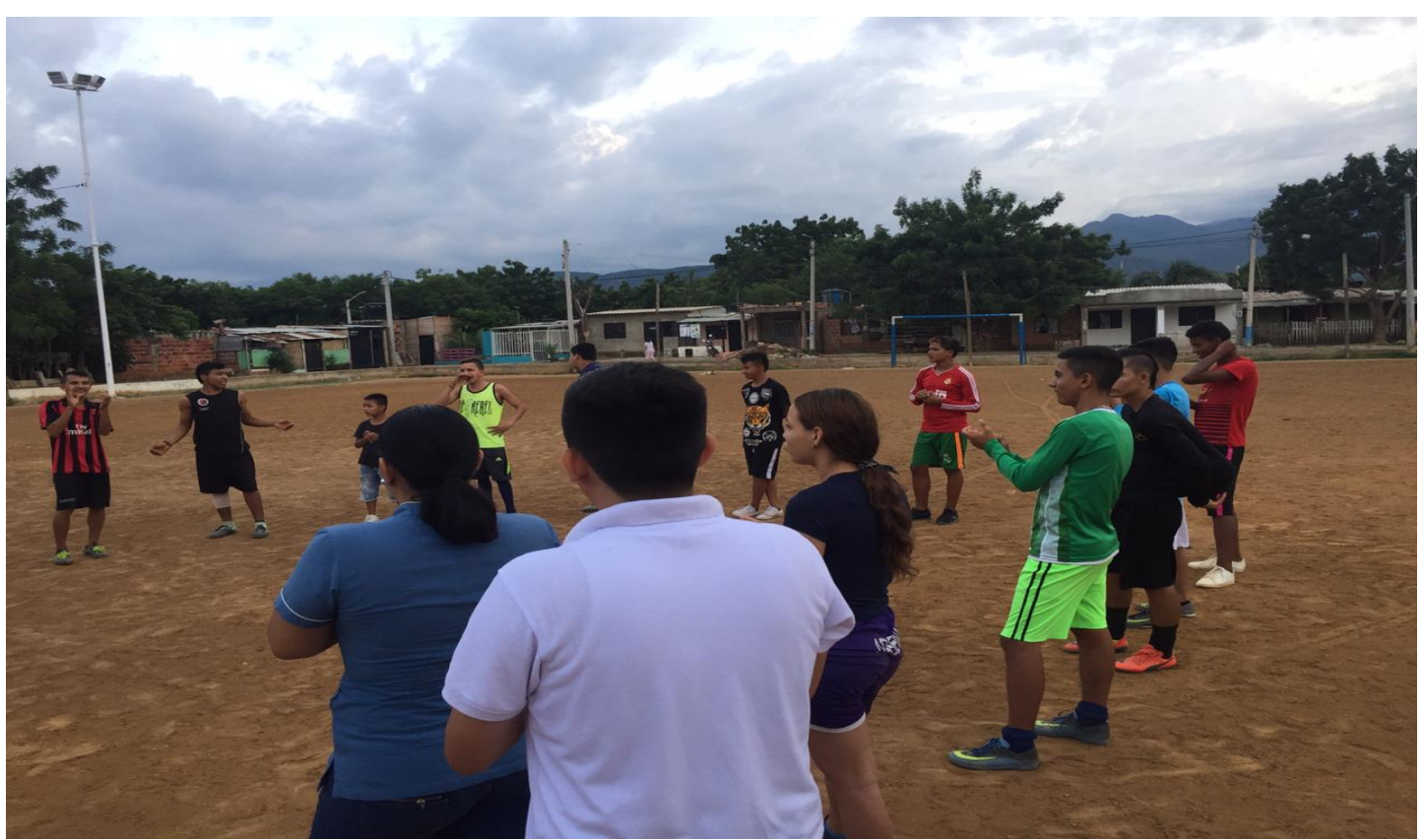

Fuente: Ramírez, 2019.

Con el fin que desde la Escuela Popular Jaime Garzón se visibilice todo lo que hacen y han hecho desde el trabajo comunitario a partir de los aportes de la comunicación popular, se han organizado y gestado espacios académicos, foros, conversatorios de participación abierta dentro de la universidad para que integrantes de la escuela y de otros procesos barriales vayan y compartan su experiencia. Tales espacios han sido bien recibidos no solo por parte de los estudiantes, docentes y directivas de la Universidad Simón Bolívar, sino también por parte de la comunidad cucuteña, pues mediante estos encuentros se aporta al enriquecimiento de otros procesos sociales y comunitarios.

De acuerdo con lo que se ha avanzado desde la intervención social en el marco de este año y medio que llevamos trabajando en conjunto con los integrantes de este colectivo comunicacional, varios de estos jóvenes han manifestado en reiteradas ocasiones su interés por estudiar Trabajo Social, como una forma de aportar a su territorio, teniéndose en cuenta el sinnúmero de problemáticas sociales, políticas y económicas que existen en la cotidianidad de la periferia y que es necesario también enfrentar desde el ámbito profesional para avanzar en la construcción de una sociedad digna y justa. 
Por medio de la gestión que se realiza con la Fundación Panamericana para el Desarrollo, se logró que a través del proyecto denominado "Jóvenes construyendo paz en los territorios" se financie la compra de indumentaria (uniformes, balones, petos, conos), para los integrantes del equipo de fútbol Atlético Garzón, dado que se carecía de los mismos y no contaban con el material necesario para sus entrenamientos y tampoco con el dinero requerido para su compra. Dicha inversión fue de alrededor de $\$ 6.000 .000$, con lo que ha sido posible seguir aportándole a este proyecto comunitario más allá de lo profesional y lo político. Se espera que desde la Escuela Jaime Garzón, además de que siga potenciando la comunicación popular, denunciando las problemáticas presentes en los barrios no solo de la periferia sino a nivel ciudad, continúen fortaleciendo las relaciones interpersonales, construyéndose desde la diferencia y haciendo uso de las diversas capacidades tanto individuales como colectivas que han sido identificadas y también fortalecidas a partir de este proceso de intervención comunitaria. El proceso comunitario que se adelanta con la Escuela Jaime Garzón ha sido conocido por parte de varias instituciones tanto a nivel local como nacional. Nada más en mayo del 2019 se contó con la visita de estudiantes y docentes de Trabajo Social de la Universidad Externado de Colombia, tal como se observa en la siguiente figura.

Figura 5. Encuentro grupal de estudiantes y docentes de la Universidad Externado de Colombia con integrantes de la Escuela Alternativa Jaime Garzón. Mayo de 2019.

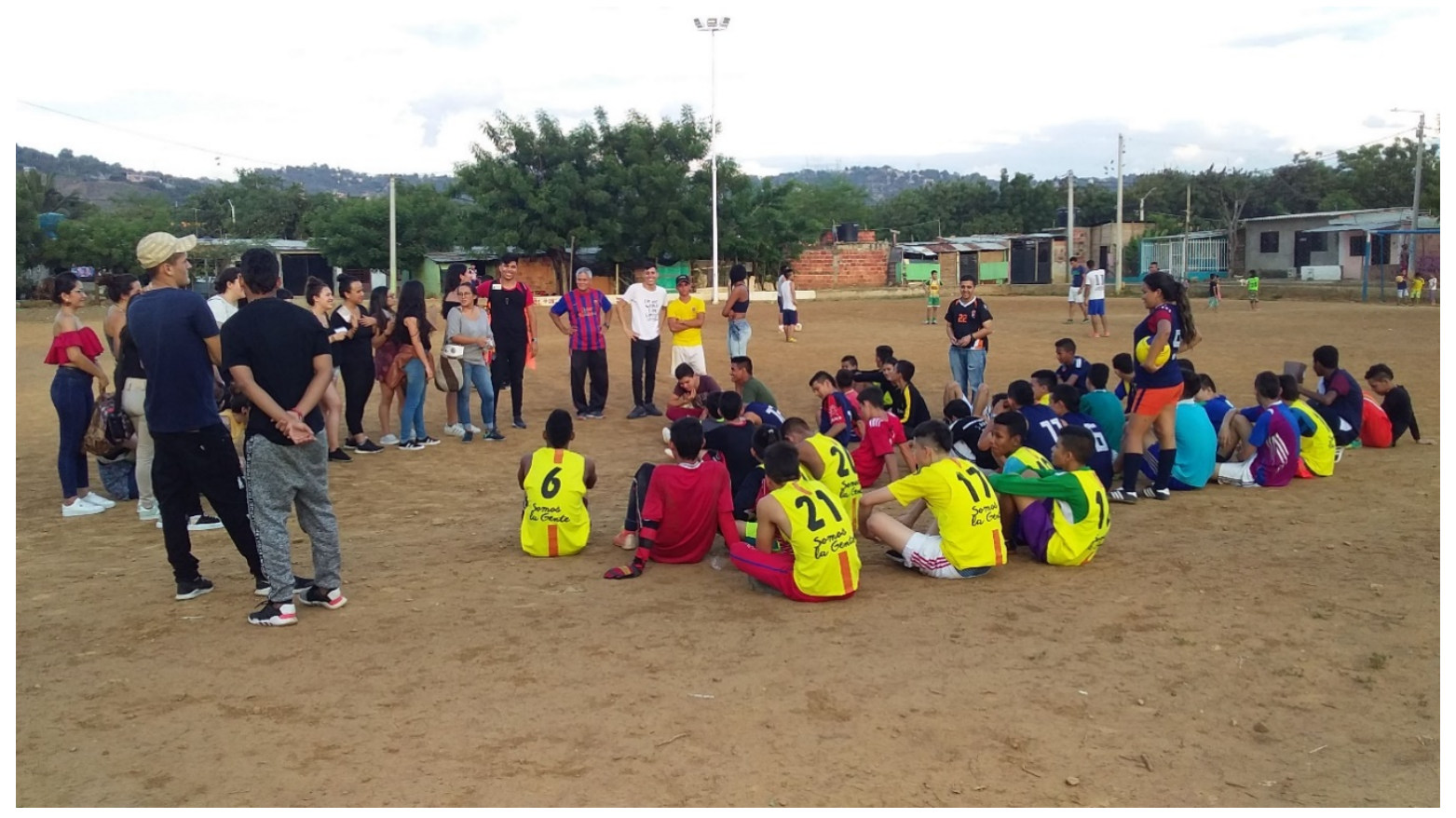

Fuente: elaboración propia. 


\section{Hallazgos}

El trabajo comunitario siempre termina por darnos lecciones a aquellos profesionales que estamos inmersos en el ámbito de la intervención social, teniéndose en cuenta la complejidad y contradicciones que se presentan en los territorios. El fortalecimiento de los vínculos se constituía en condición esencial para potenciar la organización y trabajo colectivo de los integrantes de la Escuela Popular de Comunicación Alternativa Jaime Garzón, pues no puede haber acción colectiva si existen aspectos que imposibiliten la participación, el respeto y la construcción desde las diferencias, dado que la organización comunitaria debe encontrarse relacionada con la constitución de identidad como grupo; la acción colectiva entendida de acuerdo a Melucci (1999) como ese momento en donde "los individuos, actuando conjuntamente, construyen su acción mediante inversiones "organizadas"; esto es, definen en términos cognoscitivos, afectivos y relacionales para darle sentido al "estar juntos" y a los fines que persiguen" (p.38). Con el proceso que se había implementado se estaba contribuyendo a la gestación de un horizonte de solidaridad, de trabajo en equipo, tan trascendental en el espacio social y político para quienes le apostaban a la organización social desde el compromiso ético-político.

En la medida en que avanzaba el proceso de acompañamiento de nosotros como profesionales, las personas de la escuela dejaban entrever la importancia que tenía para ellos el construir una propuesta comunicacional en donde el interés central sea la configuración colectiva de una comunicación popular que se enmarque en la visibilización de su realidad y en la defensa de los intereses de esas comunidades, de las que ellos también forman parte y en donde el acceso a los derechos sociales se constituía en un limitante. En ese orden de ideas, la comunicación popular:

\footnotetext{
Se constituye en oportunidad real para hacer visibles los procesos organizativos, pero también para visibilizar las experiencias que se adelantan por parte de los profesionales en y con las comunidades, posibilitando a la vez la formación, politización, organización y movilización social; se constituye en opción para fomentar la identidad y la diversidad cultural, generando espacios de diálogo y de acción entre los sujetos para avanzar en la construcción de propuestas colectivas, siendo así mismo estrategia de resistencia o de oposición al poder hegemónico. (LassoUrbano, 2019, p. 162)
}

Así que era necesario construir conjuntamente con los pobladores de estos barrios alternativas liberadoras, basadas en ideas de igualdad y comunidad, de manera que el trabajo colectivo, articulado, participativo, debía trascender más allá del escenario de la escuela. La acción colectiva debía concebirse en el aspecto orientador para la búsqueda y el fortalecimiento de la organización, por medio de la creación de agendas conjuntas, las cuales sean discutidas y consensuadas, en las que se integren los diversos puntos de vista de los participantes, lo que se constituye en una apuesta por articular intereses comunes desde la diversidad. 
Todo proceso de organización social, implica necesariamente la generación o potenciación de lazos, de camaradería, de respeto, de confianza, de solidaridad, de construcción colectiva. En todo proceso de comunicación popular los parámetros manifestados también tienen que estar presentes no sólo entre los comunicadores populares, sino además en relación con la comunidad y en este proyecto comunicacional no sería la excepción, a partir de la interacción permanente que se estaba teniendo los vínculos fueron afianzándose. A partir de los postulados de Antonio Gramsci, la organización social es entendida como la acción colectiva que emprenden los distintos sectores populares, encaminada hacia las luchas contra las formas de poder, represión y discriminación, principalmente en la vida cotidiana, siendo además una forma de resistencia ante la hegemonía y la dominación. Ésta, implica que se asuman procesos constantes de formación y preparación ideológica por parte de aquellos que hacen parte de la misma, por lo tanto, dentro de la organización social se hace fundamental: "reforzar ideológica y políticamente a los individuos que la integran, para así de ese modo hacerlos capaces de contener y encuadrar masas aún más amplias sin que la organización sufra demasiadas sacudidas" (Gramsci, 1967, p. 23); esta debe convertirse en elemento esencial para comprender y reconocer las dinámicas de la realidad concreta y así se logre asumir acciones colectivas que aporten a la transformación social de la misma.

Una vez como grupo identifican las capacidades con que se cuenta tanto de forma individual como colectiva, se reconocen las problemáticas presentes, las necesidades existentes, los elementos que afectaban la dinámica de la Escuela Jaime Garzón; en conjunto con el profesional social y el grupo de estudiantes de Trabajo Social se comienza a avanzar en su interpretación y en el diseño de la estrategia metodológica donde cada integrante de la escuela tendría un rol protagónico tanto en la construcción e implementación de la propuesta de intervención comunitaria.

La escuela no podía ser concebida simplemente como un lugar a donde se llega a escuchar a expertos en alguna temática en específico, a hacer parte del debate académicopolítico, a aprender de contenidos para apropiarlos en la comprensión y abordaje praxiológico de la realidad concreta, a pensarse propuestas colectivas comunicacionales que involucren la participación de las comunidades, sino que también se necesitaba dotarla de humanidad. Muy pertinentes resultan los aportes de José Martí (2003) cuando expresa "lo que han de llevar los maestros por los campos: no solo explicaciones agrícolas e instrumentos mecánicos, sino la ternura, que hace tanta falta y tanto bien a los hombres" (p. 3), así que se debía posibilitar la comprensión frente a las dinámicas de la sociedad y la construcción de individuos íntegros, fundados en la reciprocidad, en la capacidad de admiración, en el generar vínculos afectivos no solamente entre quienes hacen parte de la organización, sino también con los mismos habitantes del territorio con quienes se comparten sueños, esperanzas, la construcción de una sociedad más digna y justa. 
Necesitaban del apoyo de personas que comprendan sus problemas y tristezas, que escuchen sus inconformidades, que sepan escucharles, antes de juzgarles.

Por otro lado, pensarse la articulación entre lo objetivo y lo subjetivo se constituyó en otro aspecto esencial en el marco de esta intervención profesional, pues muchos de los problemas subjetivos y máxime en los sectores urbano-populares surgen a partir de las condiciones objetivas. No podemos entender las relaciones conflictivas, el irrespeto, la intolerancia, si no entendemos el contexto en el que habitan los sujetos, a quienes constantemente se les violan sus derechos y que además carecen de condiciones materiales que garanticen una buena calidad de vida, como señala Fals-Borda (2009) "Lo material incluye no sólo lo constatable de la naturaleza sino también las condiciones fundamentales, primarias, de la existencia humana" (p. 256). Así que las comunidades deben comprenderse desde una perspectiva de totalidad, de lo simple, lo confuso, lo humano. Uno de los grandes aciertos de este proceso fue conocer su cotidianidad profundamente, sus visiones de mundo, darse el tiempo para hablar con ellos, no caer en la inmediatez, ni en la fragmentación de su realidad, eso se comenzó a hacer una vez me acerco al proceso y cuando ya llegan los estudiantes de Trabajo Social a contribuir en dar respuestas desde el ejercicio profesional a unas necesidades determinadas, ya existía un camino que se había empezado a trazar, pero se entendía que sus aportes eran determinantes para darle el direccionamiento requerido.

Por su parte, la interpretación simbólica de los espacios del barrio, de su historia, su gente, del territorio en general, para llenarlos de contenidos y valores que reflejen su idiosincrasia e identidad para que sean apropiados por sus habitantes e incluso por los miembros de la Escuela Jaime Garzón, fue un acierto importante que se logró hacer con el programa radial "Hablamos de la comuna", pues no solo posibilitó comprender las formas en que se organizan las comunidades, sino además sus historias, sus problemáticas, también las capacidades con que cuentan, ya que para ello se hacía necesario "extraer los materiales de la propia vida, de la vida que se pretende cambiar a través de la lucha en los planos más diversos. Hay que huir de toda condición superflua" (Mazzeo et al., 2007, pp. 14-15).

El estar comprometidos con un proyecto significa no solamente reconocer las virtudes o avances, también vislumbrar las limitaciones, los errores que se comenten, las contradicciones internas y los aspectos que afectan la dinámica grupal, y, por ende, el proceso como tal en su estructura, en su esencia; los integrantes de la Escuela Jaime Garzón han comprendido que es necesario avanzar en la unidad desde la diversidad, la diferencia, la cual implica la organización y movilización como colectivo. Los aportes del proceso de intervención en ese sentido fueron muy relevantes para potenciar el diálogo y los vínculos intergrupales y enriquecer el trabajo colectivo. La diversidad sociocultural de quienes integran la escuela es un aspecto que también contribuye a que se presenten los problemas al interior del grupo, dado que muchos provienen de otras regiones, por lo que esta comunidad no es homogénea, partimos por comprender esto, además, los conflictos sociales 
siempre van a estar presentes dado que es una condición necesaria de la sociedad lo que se requiere es idear estrategias para gestionarlos.

A medida que iban pasando los encuentros la confianza hacía su aparición, las diferencias, se estaban convirtiendo en la razón para comenzar a hacer juntos, sabían que el trabajo colectivo dependía de todos, puesto que había cosas para aprender de aquello que se estaba presentando y por ende se tenían elementos también para aportar. La escuela se veía cada vez más como un hogar, donde los integrantes se hacían una familia tal como señala uno de los jóvenes:

La Escuela Popular de Comunicación Alternativa es un espacio donde se nos da a nosotros la oportunidad de expresarnos, de decir aquellas dudas que tenemos, donde podemos reflexionar aquellas problemáticas que tenemos en nuestro barrio, en nuestro país, es un espacio en el que somos una familia. (Andrés, 15 de mayo de 2020)

La participación estaba ahí, lo que se hizo fue aportar a su fortalecimiento, a que cada integrante de la escuela se conciba como sujeto colectivo, a potenciar capacidades e identificar otras, a afianzar su identidad como escuela. Para avanzar en la comprensión del proceso, se requería pensarse en la comprensión de conceptos generales como organización, participación, acción colectiva, para ellos fue necesario analizar su esencia, su significado y la forma en que estos se entendían por parte de los integrantes de la escuela tanto de forma individual como colectiva; eso fue lo que se hizo, el compromiso como profesionales ante las exigencias de la realidad nos invita a no caer en su abstracción, sino que "es precisamente la "lectura del mundo" la que va permitiendo el desciframiento cada vez más crítico de la o de las "situaciones límite", más allá de las cuales se encuentra lo "inédito viable". (Freire, 1992, p. 132), poniéndose la profesión al servicio de las mayorías.

Ahora bien, en año y medio de estar inmerso aportando en el proceso comunitario se logra observar cómo la escuela también se ha había constituido de forma clara en un escenario de formación, de debate político, donde pensarse cómo contribuir a la construcción de una sociedad digna y justa se hacía algo necesario, como lo señala uno de los integrantes en su relato:

La Escuela Jaime Garzón es un proyecto de vida para el joven, en donde éste se pueda preguntar qué es lo que quiere hacer en un futuro, encuentre una salida a esta sociedad a veces tan caótica, de tanto laberinto, que no encuentra, qué hay, dónde, por dónde; entonces la escuela trata de ser como este proyecto de vida que ayuda a que el joven se deslumbre, a qué es lo que quiere hacer más adelante con su vida, siga usted. Yo creo que defino la escuela como un espacio, como una casa sin fronteras, que no está limitada, que busca sobre todo que chicos, adolescentes, jóvenes se puedan acercar puedan aprender también un poco sobre comunicación popular alternativa, que puedan, digamos, tener ideas propias y ser críticos, ser muy pilosos. (Mauricio, 15 de mayo de 2020) 
Lasso-Urbano

Es decir que se había logrado aportar a que la escuela tuviese un valor más significativo para cada integrante, que sea un espacio desde donde se puede pensar el barrio, idear estrategias conjuntas para contribuir a la generación de cambios desde lo individual y lo colectivo, sin dejar de lado claro está, el pensar procesos de mediano y largo plazo. No obstante, es importante señalar que la comunicación popular se plantea como un espacio de participación, educación, concientización, organización y construcción de la realidad social, que desde espacios locales permite la producción, distribución y acceso a los medios de comunicación, desde y para los sectores populares; en la medida que se construye la comunicación a través de lenguajes que fortalecen la identidad, el reconocimiento de diversas expresiones culturales y la utilización de recursos. Por último, mencionar que la Escuela Popular de Comunicación Alternativa Jaime Garzón se convertía -y para orgullo de sus integrantes- en escenario a través del cual era posible apostarle a la verdadera praxis política.

\section{Conclusiones}

A partir de este proceso de intervención comunitaria y de la sistematización de la misma, son muchas las conclusiones a las que logramos llegar, entre las más significativas se encuentran las siguientes:

- El conocimiento de la realidad subjetiva y objetiva como un todo, aporta considerablemente para idear estrategias claras de intervención social, que tengan como principal apuesta desde la acción eficaz y participativa, contribuir a su transformación, pues no podemos comprender a los sujetos sociales, si dejamos de lado el análisis del contexto social, político, económico y cultural en el que se desenvuelven sus vidas.

- Para intervenir en procesos comunitarios, se necesita no solamente idear estrategias que partan de la identificación de las necesidades o problemáticas existentes, sino, además, desarrollar una planeación estratégica que se construya con las poblaciones de forma participativa, pues eso hace que los sujetos sociales se apropien de sus propuestas, potencien o identifiquen sus capacidades para más adelante, cuando los profesionales sociales ya no estén, sean ellos los encargados de direccionar este tipo de proyectos colectivos.

- El potenciar la organización y la acción colectiva también se lleva a cabo desde la resolución de conflictos, desde el diálogo, el debate colectivo, el respeto hacia las diferencias. 
- Para transformar la fragmentación grupal se necesitaba crear unidad en la diversidad, a sabiendas del contexto tan complejo en el que se está. Apoyarse como grupo implica también contar con la capacidad de construir desde la diferencia.

- Como profesionales sociales debemos abordar la experiencia desde las categorías y ejes significativos, tanto desde los actores como desde nosotros mismos para así comprender la práctica, para transformarla, para mejorarla.

\section{Referencias Bibliográficas}

Bourdieu, P. (2002), El oficio del sociólogo. Buenos Aires, Argentina: Siglo XXI Editores.

Fals-Borda, O. (2009). Una sociología sentipensante para América Latina. Bogotá, Colombia:

CLACSO; Siglo del Hombre Editores.

Freire, P. (1992). Pedagogía de la esperanza: un reencuentro con la pedagogía del oprimido. Ciudad de México: Siglo XXI Editores.

Freire, P. (2004). Pedagogía de la autonomía: saberes necesarios para la práctica educativa. Sao Paulo: Editorial Paz e Terra SA.

Gramsci, A. (1967). La Formación de los Intelectuales. México: Editorial Grijalbo S.A.

Jara-Holliday, O. (1994). Para sistematizar experiencias: una propuesta teórica y práctica. San José, Costa Rica: Editorial Alforja.

Jara-Holliday, O. (1998). El desafío político de aprender de nuestras prácticas. San José, Costa Rica: Editorial Alforja.

Jara-Holliday, O. (2018). La sistematización de experiencias: práctica y teoría para otros mundos posibles. Bogotá, Colombia: Cinde.

Lasso-Urbano, C. (2019). La comunicación popular como escenario de praxis para el Trabajo Social Comunitario. Eleuthera, (21), 152-167.

Martí, J. (2003). Maestros ambulantes. Recuperado de https:/ / www.biblioteca.org.ar/libros/1139.pdf.

Mazzeo, M., Acha, O., Campione, D., Casas, A., Gaviasca, G., Dri, R., ... Stratta, F. (2007). Reflexiones sobre el poder popular. Buenos Aires: Editorial El Colectivo.

Melucci, A. (1999). Acción colectiva, vida cotidiana y democracia. México: El Colegio de México.

Ramírez, J. A. (Fotógrafo). (2019). Actividad. Reconociendo las problemáticas presentes en el entorno. [Fotografía]. Cúcuta, Colombia: Universidad Simón Bolívar.

Sánchez, A. (2003). Filosofía de la praxis. Buenos Aires, Argentina: Siglo XXI Editores.

Torres-Carrillo, A. (2011). La interpretación en la sistematización de experiencias. Decisio, (28), 47-54. 


\section{OTROS ARTÍCULOS DE PROSPECTIVA No. 31 DE 2021}

\section{PRESENTACIÓN}

Presentación. Reflexiones sobre desafios al publicar sistematizaciones

Rosa María Cifuentes-Gil

\section{EDITORIAL}

Reflexiones sobre Trabajo Social: aportes de la Sistematización

María Rocío Cifuentes-Patiño

\section{ARTÍCULOS}

Hacer lo que se sabe, pensar lo que se hace. La sistematización como modalidad investigativa Alfonso Torres-Carrillo

Aportes y desafios de la Sistematización de experiencias en el Trabajo Social y la extensión crítica. Apuntes y reflexiones desde la perspectiva de la Educación Popular

María Rosa Goldar

Valeria Chiavetta

La sistematización en Trabajo Social y la epistemología feminista del punto de vista. Diálogos sobre la producción de conocimiento sustentada en experiencias

Ruth Noemí Parola

María Florencia Linardelli

La Sistematización investigativa de las experiencias: del baile de los que sobran a la fiesta de los que faltan

María Belén Ortega-Senet

Sistematización y Trabajo Social en Chile. El largo y sinuoso camino

Patricia Lorena Castañeda-Meneses

Ana María Salamé-Coulon
Sentipensar la pandemia COVID-19 desde la sistematización de la experiencia en Trabajo Social: reflexiones del profesor Oscar Jara Holliday

Elia Sepúlveda-Hernández

La sistematización de experiencias, una investigación social cualitativa que potencia buenas prácticas de convivencia y gobierno. La experiencia de un conjunto residencial multifamiliar en Cali, Colombia Martha Lucia Echeverry-Velásquez Manuela Prada-Dávila

Construcción de subjetividades epistemológicaspolíticas de profesoras y profesores de Investigación social en una universidad privada y confesional en Bogotá

Giovanni Mora-Lemus

Sistematización de la experiencia Reconocimiento de los derechos humanos del adulto mayor en dos familias residentes en Cali y Valledupar (Colombia)

Lina María Cuello-Lacouture

Jimena del Pilar Jaramillo-Jaramillo

La memoria transformadora como estrategia de intervención profesional en los procesos de reconciliación social: comprensión a partir de mujeres campesinas, excombatientes y jóvenes en Manizales, Colombia

Yeimmy Stephania Corredor-Sotelo

Juliana Fuertes-Fuertes

Sistematización de una estrategia de educación informal implementada en personas privadas de la libertad en el establecimiento penitenciario de mediana seguridad y carcelario de Barranquilla, Colombia

Rafael Humberto Herrera-Mercado Rafael Alberto Zambrano-Vanegas 
Aportes significativos del proceso de intervención comunitaria con la Escuela Popular de Comunicación Alternativa Jaime Garzón de la ciudad de Cúcuta, Colombia

Carlos Lasso-Urbano

La sistematización de la intervención como metodología de investigación en Trabajo Social. Importancia práctica y teórica de la fase de recogida de datos en la intervención social según experiencia del Programa de Apoyo a las Familias en Zaragoza, España

Elisa Esteban-Carbonell

Nuria Del Olmo-Vicén

Papel de la sistematización de experiencias en los procesos de evaluación de intervenciones de salud pública en la Comuna Saludable por la Paz, Cali - Colombia

Jenny Faisury Peña-Varón

Paola Andrea Marín-Velásquez

Janeth Mosquera-Becerra

Experiencia de intervención social en hogares comunitarios integrales del barrio Alfonso Bonilla Aragón, Cali - Colombia

Julián Alexander Montaño-Cárdenas

Las políticas sociales y el gobierno de la "población indígena". Estrategias y regulaciones en el multiculturalismo chileno

Rodrigo Agustín Navarrete-Saavedra

Representaciones sociales sobre estilo de autoridad y tipos de interacción en cuidadores de residencias de protección infantil en Chile

Marcelo Gallegos-Fuentes
Carmen Gloria Jarpa-Arriagada

Reflexiones sobre inseguridad social y cuestiones penales. Una respuesta estratégica a partir de experiencias de cooperativismo con ex detenidos en Argentina

Analia Elizabeth Otero

Yael Yanina Barrera

Desarrollo y salud: la emergencia de un nuevo paradigma

Jesús María Sánchez-Ordóñez

Trabajo Social en ejercicio libre: la perspectiva profesional en España

Paula Frieiro-Padín

Tamara Fernández-Arias

Rubén González-Rodríguez

\section{RESEÑAS}

Social Work and the City: Urban Themes in 21stCentury Social Work

Felipe Saravia-Cortés

Respuestas del Trabajo Social ante emergencias sociales y problemáticas sociales complejas de México y España

Felipe Saravia-Cortés

El feminismo, el género y la profesionalización del trabajo social en Colombia (1936-2004)

Ambar Oriana Serna-Lombo

El puño invisible. Arte, revolución y un siglo de cambios culturales

Carlos Arturo Robledo-Marín

\section{PROSPECTIVA}

No. $31 \bullet$ ene.-jun. 2021

e-ISSN: 2389-993X • Universidad del Valle 